\title{
Analysis on the Selection of Editing Points in Short Films and Its Significance
}

\author{
JiaxuanXing ${ }^{1, *}$ Guicheng Zhuang ${ }^{1}$ \\ ${ }^{1}$ School of Humanities, Jianghan University, Wuhan, Hubei 430056, China \\ *Corresponding author. Email:915081533@qq.com
}

\begin{abstract}
As one part of the post-production process of film and television, editing is not to be underestimated for the changes of the art of film and television, and even affects the communication effect of film and television works to a certain extent. Emotion editing points enable the audiences capture the main line of emotion, and the emotional value is highlighted; action editing points enable the audiences appreciate actions, and the action value is highlighted; and the rhythm editing points enable the films play in loose and tight management, and its expressive power is shown. Film and television works with good editing are of artistic characteristics and artistic appeal, and can fully mobilize the audience's desire to watch.
\end{abstract}

Keywords: Editing point, Selection, Significance.

\section{INTRODUCTION}

With the rapid development of film and television art in contemporary society, editing has always played an important role in film and television art. Film and television editing refers to a way of secondary processing of film and television works, and it is no longer to connect the two shots with each other monotonously, but to master the overall structure of film and television works, select the shots with a sense of direction according to the director's understanding, and finally arrange them in an appropriate way to achieve the effect of good artistic appeal of film and television works[4]. Editing technology also shows the way of thinking of film and television works. Successful film and television works have their unique editing techniques and expression techniques. Editing point is a term that often appears in post-editing. Generally speaking, it is the time to switch shots. How to select editing points depends on the specific film characteristics and conditions. Specifically, editing points can be divided into action editing points, rhythm editing points and emotion editing points. The selection of different types of editing points will directly affect film and television effects and bring different viewing experiences to the audience[3]. Besides, editing points play a unique and important role in film and television works, especially in showing the character's psychology, controlling the film rhythm, enhancing the artistic appeal, mobilizing the audience's emotion, and stimulating the audience's desire to watch.

\section{EMOTION EDITING POINT: HIGHLIGHTING EMOTIONAL VALUE}

The emotion editing points in the films are generally selected according to the inner state of the characters and the characteristics of shot modeling, with the actual emotions of the characters in various situations as the reference. The specific application of emotion editing points is in opera films and musical films. At the same time, the specific editing points should be selected in combination with the melody, rhythm and paragraph of the music. In some films with storylines, the processing of emotion editing points is to extend relevant scenes, but not to shorten them. For example, in a scene of "Majiang Battle", the protagonist exercised his power to dismiss Xia Kangbi's official position and subdued the governor. At that time, he had a bright mood and looked at the scenery in the garden, which was a long process and shot at a distance. As the lens moved, audiences would see the scene that the hero stood on the steps and looked at the distant scenery. After 
the hero image processing, the lens was not switched immediately, but the extension must be processed on site, so that the audience would feel his comfortable mood and inner state from hero's facial expression. In addition, the screen did not play either language nor body action singly. The character's inner action was still continuing and the same was for mood. If the editing point of the shots was in language or form, the inner state of the characters could not be fully displayed, the audience's emotions would be affected, and the artistic effect would not be well displayed. Therefore, in the processing of this scene, editing point was selected according to the progress of emotions, which not only aroused the audience's emotions, but also showed the artistic effect of the film.

Generally, psychological behaviors reflect the emotions of the characters. Based on this, emotion editing points in film and television works are selected according to the form of characters in different episodes. Finally, emotion editing points are determined based on the modelling characteristics of these shots. In general, the emotion editing shot length is determined by the plot requirements of the film or television works, with some scope for choice. Also, it is not limited by other actions inside or outside the frame, but mainly creates the atmosphere of the subject and the whole frame, and expresses the inner emotions and activities of the characters. The selection of emotion editing points is more difficult than that of physical action and action editing points. As long as the contents and meanings are grasped, action and emotion editing points are based on the mental activity and emotion of the characters[5]. Therefore, film cutters are required to have a deep understanding of the character's personality and mental activity.

In addition, one of the important reference factors for editing is the psychological mood of the characters. Especially for emotional films, the psychological mood of the characters is even more important. Mature editing will not only harmonize the relationship between the characters and the film well, but also show and reproduce the inner state of the characters perfectly, giving the audience a psychological and aesthetic feeling. In film and television works, emotions play a very important role. In addition to the actors' own infectious expression, emotion editing points also play a role, which should not be underestimated. A good selection of emotion editing points enables the audience to immerse into the film's emotions, at the same time, audiences will be moved by the emotions in the film and television works, so as to achieve emotional empathy.

When selecting emotion editing points, it is required to focus on the exaggeration and rendering of the characters' emotions, and take a full-length shot as much as possible with the principle of "perferring to be long rather than be short". Besides, emotion editing points have its own characteristics. Meanwhile, it also has a great deal of scope for the selection of inner emotions, and it is not limited to the external actions of the characters in the scene, but mainly depicts their internal activities, rendering emotions and creating atmosphere. For example, in "Contratiempo", a journalist interviewed a criminal who talked about his remorse for his crimes and how much he missed his loved ones at home, and when the conversation stopped, it reached the peak of emotion. The film cutters prolonged the scene deliberately. Two seconds after the offender finished speaking, he burst into tears and sobbed sadly. Then he stopped sobbing. When the character's emotional expression basically finished, film cutters would select editing point, so that the character's psychological activities would be performed incisively and vividly[6]. Finally, the audience's emotions would be mobilized and the impact of the whole film would be enhanced. If the scene after the talking was cut, it was impossible to fully convey the self-remorse of the criminal.

\section{ACTION EDITING POINTS: HIGHLIGHTING THE VALUE OF ACTION}

In the film and television editing, action editing points are usually based on the action of the subject, and should be selected according to the film and television plots and the specific environment in which the characters are in. For example, in daily life, people's sitting seems to be an uninterrupted process and action, but if photographers shoot it through the camera and decompose this action, it is found that this action is not continuous; there is a short pause; and this momentary pause is the editing point, which can be used to connect the actions. Meanwhile, it is necessary to pay attention to use up the one or two frames of that pause, and start from the first frame after the pause. Then, the action is smooth and the scene continues, and the audience would not feel any discomfort when watching. 
When photographers use different shooting scales to show an action, the first method is to connect the first shot in which the actor's action is in micro-motion, i.e. the action is about to be made, with the next shot in which the action is completed in its entirety, the sense of movement can compensate for the jump in the shooting scales and make it more fluid visually. For example, shot 1 is a close up of $\mathrm{A}$ and $\mathrm{B}$. A swings a punch at B - B falls; shot 2 is the same, but it is a panoramic view of 2 people, and it would be more effective to do the editing point at the moment when $\mathrm{A}$ hits $\mathrm{B}$, and this point is the climax of the action. In this way, the editing is both visually aesthetic and in line with the camera articulation. The second method is to have the actor's action complete a quarter of the overall action in the first shot and complete three quarters of the overall action in the next shot. However, it is suggested to be aware of the visual retention of people's eyes. There are jumps of 2 or 3 frames between the pause of the first action shot and the completion shot of the next action. After these 2 or 3 frames have been cut, there is no visual jump. For example, the video editing of the running mice in "Hollow Man" is very smooth. The film cutters did not "cut" the scenes before and after the editing point in traditional sense, and there was always a transition of one or two frames, which could be seen as a superimposition of the two frames or as a fading in and out of the two frames. From the first frame, there was a relative pause in the action, which would be smooth for the audience. In other words, when viewing frame by frame, it is found that there are relatively still frames, and this is usually the point at which film cutters choose to cut. Generally, film cutters would leave these still frames (1-2 frames) in the previous shot, and the next would move in a different scene.

The selection of action editing points is a common artistic technique in film and television works. Usually, it is based on the characters in the work and the plot of the film and television productions. In a given situation, action editing points are determined according to the actions of the subject and the laws of the subject's activities in real life. For example, when the work is accompanied by background music, the coordination between the venue and the music, the main movement and the rhythm of the music should be taken into account in the selection of the action editing points. At the same time, the selection of the editing points should be based on the required picture composition factors. In terms of actions of film and television works, for example, two men were fighting against each other. In this process, two were standing opposite each other, which was the first panorama shot. And the second shot was a close shot, with the figure flying out on the ground. Among the footage, there were two action shots from different perspectives and different scenes. Therefore, when editing this continuous action, the 'fall' should be used as the editing point for the action.

Another method of action editing is to emphasize an action by purposefully repeating it, which is known as double or triple action. The shot of raising the arms to its highest point was used as a editing point, connecting action shot taken from the front and the back. And then, this shot was linked with a full shot of the hand retracting downwards taken from the side after playing the ball. For example, in "Infernal Affairs", the motion of Tony Leung raising his arm to pull out a gun was used as an editing point, connecting the action shot taken from different angles and views. And then, this shot was linked with a large panoramic shot. In this way, the action might not be as smooth to the viewer, but the action of "pulling out the gun" was repeated three times (triple action), which not only highlighted and portrayed the action of Tony Leung raising his arm to pull out the gun, but also increased the expressiveness of the action and made a strong impression. In the whole film and television work, if the video is not completed according to the content needed by the director, the work presented to the audience must fail to achieve the expected effect. Therefore, the selection of film cutter is important. It is necessary to pay attention to the artistic cultivation of the film cutter. At the same time, the film cutters must have a sensitive insight, relevant work experience, profound understanding and advanced editing skills.

\section{RHYTHM EDITING POINTS: HIGHLIGHTING THE EXPRESSIVE FORCE OF THE FILM}

In the film and television works, the rhythm editing point refers to the shots without dialogue. According to the plot and plot rhythm in the feature film, the relationship between the characters and the task in a particular situation, and the factors such as the plot, dialogue, physical action, the shot length would be determined.

Rhythm is also an important reference factor in film and television editing. The pace of a film and television work has a huge impact on its overall 
style, and some works even infect the audience's emotions relying on rhythm completely. For short films, rhythm is an important factor that should not be ignored, and the selection of rhythm editing points is even more important. A good selection of rhythm editing points would not only promote the communication and impact of the film, but also make the viewer's experience smoother and more comfortable.

The rhythm of a film can be divided into internal and external rhythms. Internal rhythm refers to the contradictions in the plot and the changes within the characters[1]. External rhythm refers to the switching of shots, etc. In addition to the overall rhythm of the film, experienced film cutters know that each sequence and scene must also have a pace to match and be controlled according to the mood of the characters, their movements and the composition of the picture to ensure that each scene is expressed in its entirety.

The selection of rhythm editing points is the most common artistic technique used in film and television works. The selection of rhythm editing points is particularly important in productions with crowd scenes, battle scenes or transitions. Sound is essential in film and television productions[2]. When selecting film editing points, it is necessary to carefully scrutinise sound to meet the requirements of synchronisation, so that the two are accurately combined. For example, the documentary "A Bite of China" is about the traditional and local Chinese cuisine. Each episode lasts about 50 minutes and most of the footage is shot about the dishes. However, the movements of pushing, pulling, shaking and moving during filming slowed the rhythm of camera movement throughout, which was up to a few hours. Due to the time limit and the commentary limit, the whole process of food production could not be shown. In the food production process, the part of moving shot would not be used, and only fixed frame and model were used to match high-speed switch, obtaining the "process" consistent with the commentary, resulting in dazzling artistic effect, highlighting the theme, and showing a strong sense of rhythm.

Different types of films and different parts of the same film have different requirements for rhythm, and rhythm plays different roles, one of which is to highlight the different character traits and charms of the characters through the selection of rhythmic editing points. For example, in the first episode of the third season of the CCTV documentary "Heritage" — "the Belonging", Dareli Khan, the father, hoped that his son would inherit his craft of building yurt, but Songhar, the son, was not interested and distracted, and Songhar did not agree with his father's arrangements for his marriage. Meanwhile, Songhar left home, at which point the pace of the film was fast and intense, with shorter shots and smaller scenes. Besides, the character personalities were performed through inner state of characters and scenes of the film, which constituted the inner rhythm of films. At the same time, the rebellious Songkhar and the mature and silent Dahleli Khan were performed on the scene[2]. As the documentary timeline progressed, Songkhar gradually understood his father's intentions and was less rebellious, while his father was overjoyed at his son's return, and the relationship between father and son was gradually improved, the rhythm of the documentary also changed with the content of the documentary, becoming softer. At the same time, the shots were longer, the scenes were larger, and there were more panoramic views and distant views, showing another side of Songkhar and unspeakable image of his father.

\section{CONCLUSION}

Video editing is an essential and important part of film and television art. In film and television works, it plays an irreplaceable role and even determines the effect of the communication of film and television works to a certain extent. With the good use of good editing, film and television works will be of more artistic characteristics and artistic impact, and can fully mobilize the audience's desire to watch, etc. However, with the bad use of editing, people would feel awkward, and the viewing experience is impaired. Therefore, the importance of editing can be seen. Film and television technology is constantly changing and developing, and film and television works are gradually diversified. With the progress of science and technology, people's thinking of appreciation has also changed. And they put forward higher requirements for editing technology. Therefore, film cutters should adapt to the changes in the new environment, and strengthen the study on theoretical knowledge of editing, apply theoretical knowledge to practice, and strive to absorb new knowledge to improve their editing ability, so as to edit more and better film and television works. 


\section{AUTHORS' CONTRIBUTIONS}

Jiaxuan Xing wrote the manuscript, and Guicheng Zhuang revised and edited the manuscript.

\section{REFERENCES}

[1] Hong Xuefei. Study on the artistry of documentary editing from "A Bite of China 2" [J], 2014(9). (in Chinese)

[2] Liao Shangjie. Exploration of documentary editing methods [J], Public Channel of Hainan Radio and Television Station, 5079(3). (in Chinese)

[3] Tan Meiyu. The importance of post-editing of documentaries [J], Tangshan Radio and Television Station, 2012 (7). (in Chinese)

[4] $\mathrm{Li}$ Bin. Discussion on the control of the televised documentary editing [J], Jilin Radio and Television Station, 2018 (8). (in Chinese)

[5] Tan Wenjin. Exploring the choice of editing points in film and television works [J], Shenyang University, 5079 (6). (in Chinese)

[6] Zhao Wenting. On the core role of rhythm processing in film and television editing $[\mathrm{J}]$, Jilin Universities of the Arts, 6708 (9). (in Chinese) 